\title{
A PRIMEIRA LEI DA TERMODINÂMICA DOS PROCESSOS HOMOGÊNEOS
}

\author{
Alessandro Ranulfo Lima Nery e Adalberto Bono Maurizio Sacchi Bassi* \\ Instituto de Química, Universidade Estadual de Campinas, CP 6154, 13084-862 Campinas - SP, Brasil
}

Recebido em 6/1/08; aceito em 25/8/08; publicado na web em 12/2/09

\begin{abstract}
THE FIRST LAW OF HOMOGENEOUS PROCESSES THERMODYNAMICS. Thermodynamics of homogeneous processes, which corresponds to the very special situation in thermodynamics of continuous media, is used to discuss the first law. An important part of this work is the exposition of some typical mathematical errors, frequently found in the traditional presentation of thermodynamics. The concepts of state and process functions are discussed, as well as reverse and reversible processes, temporality and its implications on thermodynamics, energy reservoirs and symmetry. Our proposal is to present the first law by using a time dependent viewpoint coherent with mechanics and the foundations of that viewpoint.
\end{abstract}

Keywords: first law of thermodynamics; reversible processes; time dependent thermodynamics.

\section{INTRODUÇÃO}

A conservação da energia de um sistema, na ausência de alterações do momento linear total, ou do momento angular total do sistema, pode ser dada pela equação $\Delta U=Q+W$, frequentemente considerada a expressão matemática da primeira lei da termodinâmica para processo finito. Quando se supõe que o processo seja infinitesimal, diversas outras expressões matemáticas costumam ser usadas, entre as quais a igualdade $d U=d Q+d W$. Nosso objetivo é discutir a equação para o processo infinitesimal. Para isto, serão usados os instrumentos inerentes à termodinâmica dos processos homogêneos. ${ }^{1,2}$ Esta pode ser considerada uma introdução à termodinâmica dos meios contínuos, ${ }^{1-10}$ a qual é uma ciência temporal, assim como todas as demais ciências naturais.

A termodinâmica, criada na primeira quarta parte do século XIX principalmente por engenheiros, como uma ciência aplicada para se entender o funcionamento das máquinas térmicas, ${ }^{11}$ evidentemente não nasceu atemporal, já que se originou da necessidade de melhor compreender o temporal mundo físico real. Aliás, se por termodinâmica clássica for entendida a grande evolução conceitual implantada por Joule, Kelvin, Rankine, Reech e Clausius, a partir da segunda metade da década 40 e até a primeira metade da década 50 daquele século, a termodinâmica clássica será temporal. $\mathrm{O}$ enfoque atemporal tornou-se cada vez mais predominante durante as últimas quatro décadas do século XIX e, ainda quando Clausius apresentou a sua famosa desigualdade (1862), não havia o quase consenso geral a favor da atemporalidade que existiria 40 anos depois.

A atemporalidade se impôs porque, por meio deste artifício, foi mais fácil transformar a termodinâmica, de um amontoado de conceitos muitas vezes conflitantes entre $\mathrm{si}^{12}{ }^{12}$ num todo matematicamente coerente. Talvez, a maior contribuição à visão atemporal e à coerência matemática da termodinâmica se deva ao tratado de mecânica estatística clássica publicado por Gibbs em 1902, ${ }^{13}$ cuja reimpressão atual é facilmente obtenível e deveria ser leitura obrigatória para todos os que se interessam por conceituação termodinâmica. Gibbs foi um entusiasta da geometria e da lógica, que declarava ser a matemática não apenas uma linguagem, mas a linguagem por ele preferida.

Tolman, ${ }^{14} \mathrm{em}$ 1938, produziu a obra lapidar da mecânica estatística, onde se encontra tanto a estatística clássica como a quântica (os

*e-mail: bassi@iqm.unicamp.br novos livros de mecânica ou termodinâmica estatística, publicados hoje em dia, são apenas de estatística quântica, tanto porque esta é a única que fornece resultados corretos para algumas quantidades, notadamente as capacidades térmicas, como porque, de acordo com Hill, ${ }^{15}$ a estatística quântica é mais facilmente explicável). Na sua parte clássica, Tolman baseia-se nos legados de Boltzmann e Gibbs, quase transcrevendo literalmente este último, no que se refere aos conceitos fundamentais da termodinâmica estatística. Foi o rigor matemático da termodinâmica estatística de Gibbs que consolidou a transformação da termodinâmica numa teoria atemporal, quase 80 anos após Carnot.

Com isto, a termodinâmica passou a fornecer respostas precisas e utilíssimas. Mas, por outro lado, pagou o alto preço da sua atemporalidade, o que a isolou de todas as demais ciências naturais. Para o químico, é particularmente notada a separação entre termodinâmica e cinética químicas. Porém, desde que surgiu a tendência atemporal, surgiu a resistência a ela. Famoso livro didático de termodinâmica, ${ }^{16}$ publicado por Planck pela primeira vez em 1891 e reeditado em alemão e em inglês até 1921 (também pode ser facilmente obtido, em reimpressão atual), já considerava a atemporalidade um preço alto demais para ser pago por uma ciência natural. Aliás, mais de uma década antes da primeira edição do seu livro Planck defendeu, em sua tese de doutorado (1879), a necessidade de se procurar a coerência matemática na termodinâmica, mas sem abrir mão da sua temporalidade. ${ }^{17}$ Mesmo assim, o cientista alemão Planck repetidamente elogiou seu colega americano Gibbs.

Pode-se considerar que a teoria temporal denominada termodinâmica do não equilíbrio, ou dos processos irreversíveis, ${ }^{18-20}$ inicia-se em 1931, com a publicação dos trabalhos de Onsager. ${ }^{21,22}$ Entre muitos artigos que se seguiram, destacam-se os de Eckart, ${ }^{23}$ Casimir $^{24}$ e a revisão de experimentos publicada em 1960, por Miller. ${ }^{25}$ Pode-se, ainda, considerar que a termodinâmica temporal dos meios contínuos, ou racional, ou estendida, inicia-se em 1960, com o trabalho de Truesdell e Toupin. ${ }^{3}$ Aqui, também, segue-se grande quantidade de trabalhos, entre os quais os outros 9 livros já citados,,$^{1,2,4-10}$ sendo de 2002 o último deles.

Estas duas teorias diferem, entre si, principalmente no que se relaciona à exigência de linearidade para os tensores que descrevem as deformações corporais, ou seja, à exigência de que o comportamento do corpo seja uma extensão linear do seu comportamento no equilíbrio, o que restringe o estudo a regiões próximas ao equilíbrio. Tal 
exigência, inexistente na termodinâmica dos meios contínuos, torna esta teoria mais abrangente. Após uma extrema simplificação, causada pela imposição de homogeneidade espacial para os valores de todas as propriedades intensivas, a termodinâmica dos meios contínuos se reduz à termodinâmica dos processos homogêneos, que será utilizada no presente trabalho. Para o ensino da termodinâmica temporal em nível de graduação, surge em 1998 o primeiro livro didático, ${ }^{26}$ hoje já com 5 reimpressões (a última em 2006). Em 2001, Química Nova publicou um artigo onde a primeira lei é discutida usando-se termodinâmica temporal. ${ }^{27}$

Desde já, cabe esclarecer que, no presente trabalho, é usado o significado dado por Truesdell ${ }^{1}$ para a expressão "termodinâmica tradicional". Não se trata da termodinâmica de Gibbs, mas sim daquela que é costumeiramente ensinada hoje em dia, no mundo inteiro, a qual é muito diferente da teoria proposta por Gibbs. De fato, dentro da visão tradicional da termodinâmica existem variados enfoques, o que já evidencia não se tratar da visão específica de Gibbs. Recente trabalho envolvendo diversos livros didáticos em termodinâmica tradicional, entre os quais 7 que se situam entre os mundialmente mais vendidos hoje em dia, ressalta profundas diferenças conceituais entre eles. ${ }^{28}$

Aparentemente, este fato é pouco divulgado. Quem aprende termodinâmica por determinado livro didático, tende a acreditar que aquela é a única ou a verdadeira termodinâmica, como realmente acontece com outras disciplinas, onde os livros didáticos diferem entre si apenas no modo de expor a matéria e na seleção dos tópicos neles incluídos. Isto não ocorre na termodinâmica tradicional porque os autores de livros didáticos, nesta disciplina, afastam-se da termodinâmica de Gibbs, que consideram demasiadamente matemática e abstrata.

Tais autores buscam um enfoque mais próximo do dia-a-dia e da vivência do estudante, ou da hoje bem conhecida visão corpuscular dos gases, ou de experiências laboratoriais (nem sempre de fato realizáveis) para, desta forma, transmitir os conceitos de um modo que facilite a assimilação dos mesmos, em comparação com as exigências lógico-matemáticas características do enfoque de Gibbs. Mas, como a natureza é temporal, surgem dificuldades que apenas podem ser superadas mediante artifícios. É precisamente da escolha entre diferentes artifícios, de acordo com o que cada autor considere mais aceitável, que surgem as diferenças conceituais. Aliás, não apenas em livros didáticos percebem-se diferenças conceituais. Por exemplo, são bem conhecidas as diversas versões tradicionais da segunda lei da termodinâmica, que absolutamente não são modos diferentes de dizer a mesma coisa, nem versões complementares entre si. ${ }^{29}$

Como consequência, mais de um século após a publicação do trabalho de Gibbs, que consolidou a artificiosa visão atemporal em troca de rigorosa coerência matemática, a termodinâmica tradicional voltou a ser como esta ciência era antes da contribuição de Gibbs, ou seja, ela hoje contém conceitos discrepantes, por causa da adição, à imposição de atemporalidade, de uma aversão ao rigor matemático que agride a memória de Gibbs. Esta aversão geralmente é camuflada, mas, em pelo menos um caso, ela é explicitamente declarada e defendida. ${ }^{30}$ Ao invés da linguagem matemática universal em que Gibbs se apoiava, uma falsa matemática específica da termodinâmica tradicional, ingenuamente construída, é usada para, aparentemente, ligar um fato experimental ao outro. Em três dos seus livros aqui citados, ${ }^{1,2,12}$ o matemático C. A. Truesdell denuncia a matemática usada nos livros de termodinâmica tradicional. Sobre este assunto, dois textos foram disponibilizados em português, pelo segundo autor do presente trabalho, na world wide web. . $^{31,32}$

Um outro fator que concorre para a incursão em equívocos, além da imposição de atemporalidade somada à aversão ao rigor matemático, é a utilização do conceito de reservatório. Tal utilização, na termodinâmica tradicional, é muito frequente, pois se trata de um conceito que facilita a explicação das igualdades e desigualdades características desta teoria. Porém, é apenas isto, porque um reservatório é, por definição, um sistema que não obedece às mesmas leis físicas do sistema em consideração, embora com este mantenha contato físico (se obedecesse, seria apenas uma vizinhança, ou o exterior do sistema considerado).

Logo, se o sistema passasse a ser o reservatório e vice-versa, poderia não ocorrer o tipo de simetria de comportamentos que se espera aconteça sempre que os dois sistemas em contato forem regidos pelas mesmas leis. Como a escolha dos limites do sistema é completamente arbitrária, a possibilidade de quebra desta simetria é tão inatural quanto a atemporalidade. Assim como ocorre para esta última, surgem então dificuldades que apenas podem ser superadas mediante artifícios (já é um artifício tanto a atemporalidade quanto o conceito de reservatório). Novamente, é da escolha entre diferentes artifícios, de acordo com o que cada autor considere mais aceitável, que surgem as diferenças conceituais.

A termodinâmica tradicional, porém, contém numeroso conjunto de fatos experimentalmente comprovados, de tabelas e de dados utilíssimos, bem como de equações que, nas condições a elas adequadas, funcionam corretamente. Para aqueles que fazem uso prático da termodinâmica (engenheiros, pesquisadores, experimentalistas...), o que interessa são precisamente as tabelas, os dados e o seu manuseio correto, não os detalhes teóricos. Por isto, não apenas a falsa matemática da termodinâmica tradicional é exclusivamente instrumental, como a própria termodinâmica tradicional é, somente, um instrumento a ser usado em outras pesquisas. Por isto, a maneira mais apropriada de lecionar a termodinâmica tradicional é por meio da resolução de exercícios que ensinem os alunos a utilizar o que ela tem de melhor a oferecer, para o desenvolvimento da ciência e da tecnologia, sem perda de tempo com teorias inconsequentes. Porque, se alguém desejar trabalhar no desenvolvimento da teoria termodinâmica propriamente dita, deverá abandonar a chamada termodinâmica tradicional.

Não se deve supor que a existência do mencionado grande conjunto de fatos experimentalmente comprovados, de tabelas etc., indique que a matemática utilizada pela termodinâmica tradicional esteja correta. Exatamente o mesmo grande conjunto é, também, coerente com a termodinâmica dos processos irreversíveis e com a termodinâmica dos meios contínuos. Mas isto não implica em que as três teorias sejam igualmente certas e, por isto, seja possível optar por aquela que, aparentemente, é matematicamente mais fácil. A opção pela termodinâmica tradicional apenas pode ser feita quando o enfoque for estritamente instrumental, conforme colocado no parágrafo anterior.

De fato, as outras duas teorias, além dos experimentos abrangidos pela termodinâmica tradicional, também justificam experimentos adicionais, como, por exemplo, as relações recíprocas de Onsager ${ }^{21,22}$ e os experimentos citados na revisão efetuada por $\operatorname{Miller}^{25}$ (termodinâmica dos processos irreversíveis), bem como a grande quantidade de experimentos em sistemas homogêneos metaestáveis e instáveis ${ }^{33,34}$ (termodinâmica dos processos homogêneos) e os tradicionais experimentos típicos da mecânica dos fluidos, a qual, assim como a termodinâmica dos processos homogêneos, é uma parte da termodinâmica dos meios contínuos. ${ }^{1-10}$ É bem conhecida, pelos químicos, a possibilidade de que diversas teorias, certas ou não, consigam explicar os mesmos experimentos. Por exemplo, diversos mecanismos de reação podem justificar a mesma lei cinética experimental, mesmo que nenhum deles esteja quimicamente correto.

É extremamente comum o estudante de química e o seu professor não entenderem a termodinâmica tradicional, mas se envergonharem de admitir isto, porque por humildade tendem a supor que se trate de falha própria. Quando se lembra que a termodinâmica tradicional 
foi criada para facilitar o entendimento da termodinâmica de Gibbs, percebe-se que a termodinâmica tradicional é um perfeito exemplo científico do velho ditado popular "o barato sai caro". Ao longo deste trabalho ainda se cumpre uma obrigação, principalmente com o leitor, mas também com Anacleto e Anacleto, ${ }^{35}$ de responder a todas as objeções por eles levantadas ao já mencionado artigo publicado por Química Nova em 2001. ${ }^{27}$ Pretende-se, porém, fazê-lo de uma forma útil para todos os que se interessem por este importante tema, ou seja, pretende-se usar esta oportunidade para discutir vários conceitos fundamentais, relacionados com a forma diferencial da primeira lei da termodinâmica.

\section{FUNÇÃO TEMPORAL}

Considerando que a definição matemática de derivada exige que os limites à esquerda e à direita coincidam no ponto de derivação, defendem Anacleto e Anacleto ${ }^{35}$ a inexistência das derivadas temporais $d Q / d t$ e $d W / d t$. De fato, afirmam que "Como o tempo t só pode crescer, o limite quando $t^{\prime} \rightarrow t^{+}$não se pode considerar e, conseqüentemente, a derivada da hipotética função $Q(t)$ não existe. $O$ mesmo se aplica à função $W(t)$.”. Afirmam, portanto, que o limite à direita não existe porque, fisicamente, o tempo não retrocede. Sublinhe-se que Anacleto e Anacleto ${ }^{35}$ não situam a pretendida inexistência das derivadas $d Q / d t$ e $d W / d t$ em alguma característica específica do calor e do trabalho, mas sim numa característica específica do tempo. Portanto, a objeção colocada, evidentemente, seria aplicável a toda e qualquer derivada temporal, o que inviabilizaria o conceito de velocidade, entre tantos outros. Se isto fosse verdade, durante séculos gerações de matemáticos e físicos teriam incorrido em erro.

Anacleto e Anacleto ${ }^{35}$ parecem se esquecer do fato bem conhecido de que cada realidade física específica deve ser posteriormente imposta, como uma condição de contorno restritiva, a uma solução matemática mais geral, obtida por meio do cálculo diferencial e integral. Por exemplo, é exatamente porque o tempo não retrocede que se pode garantir que, se o valor de uma derivada temporal for positivo, a grandeza que estiver sendo derivada estará aumentando no momento considerado, enquanto que se o valor da derivada for negativo, tal grandeza estará diminuindo (se o tempo pudesse retroceder, o oposto também seria possível).

Mas, suponha que Anacleto e Anacleto ${ }^{35}$ se expressaram de modo equivocado. Suponha que, na verdade, eles considerem que as grandezas calor e trabalho apresentem alguma especificidade conceitual que torne impossível a derivação temporal das mesmas. Ocorre que $d W / d t$ é a costumeira potência, que pode ser volumétrica expansiva, elétrica etc.. A potência $d W / d t$ não é, na mecânica ou na eletrodinâmica, algo diferente do que é na termodinâmica. Ela é a mesma, porque as teorias são artifícios mentais por nós criados para entender a natureza, de modo incompleto e, por isto mesmo, as teorias podem diferir entre si, mas não se supõe que exista uma realidade natural diferente para cada teoria diferente.

Portanto, a potência volumétrica expansiva, ou a potência elétrica, ou qualquer outra, é sempre a mesma grandeza natural, com as mesmas características intrínsecas, independentemente da teoria em questão. Por exemplo, por mais que alguém defenda a termodinâmica tradicional, mesmo assim dificilmente esta pessoa afirmaria que a natureza é atemporal, mas apenas que o enfoque da natureza, nesta teoria, é atemporal. Analogamente, se a função $W=W(t)$ existir e for derivável em alguma ciência natural, o mesmo acontecerá em qualquer outra ciência natural. Pode-se, isto sim, pretender que a termodinâmica atemporal não utilize este fato, porque estranho à teoria considerada, mas não que o negue.

Quanto a $d Q / d t$, todo bom calorimetrista sabe que o calorímetro mede potência térmica transferida, não calor. Aliás, é bem conhecida a lei de Fourier para a transmissão condutiva de calor. Nesta lei, que geralmente pode ser encontrada nos mesmos livros didáticos de físico-química que apresentam a termodinâmica tradicional (embora em capítulos ou mesmo partes diferentes do livro), necessariamente aparece a derivada $d Q / d t$. O que já foi apresentado é suficiente como contra-argumento à afirmação de Anacleto e Anacleto. ${ }^{35}$ Mas, como este trabalho não pretende se restringir à resposta aos citados autores, deve-se aprofundar alguns aspectos matemáticos da questão.

Para isto, suponha que a função $W=W(t)$, ou $Q=Q(t)$, corresponda a elevar $t$ ao quadrado (fisicamente, isto é um absurdo). Qualquer estudante de química em nível de primeiro ano de graduação sabe que a derivada da função $x^{2}$ é a função $2 x$. Perguntado sobre isto, o estudante não responderá diferentemente de acordo com o fato de $x$ representar uma distância, um instante, uma temperatura ou uma massa, porque sabe que a resposta depende exclusivamente da forma matemática da função, logo não depende da grandeza física que a sua variável independente represente. Portanto, se a grandeza física não for derivável, a forma matemática da função que a represente já deverá refletir isto (não alguma posterior consideração adicional).

Como a existência de derivada é uma informação fornecida pela forma matemática da função, esta última estará errada se não houver coerência entre tal informação e a realidade física. Por exemplo, suponha agora que a função $W=W(t)$, ou $Q=Q(t)$, corresponda à obtenção da tangente da variável tempo, representada por $t$ (fisicamente, isto também é um absurdo). Como tan $x$ não será derivável em $x=n \pi / 2$, quando $n$ for um inteiro ímpar, esta forma da função $W$ $=W(t)$, ou $Q=Q(t)$, não será correta se, fisicamente, existir um valor da derivada para $t=n \pi / 2$, sendo $n$ um inteiro ímpar.

Suponha que Anacleto e Anacleto ${ }^{35}$ considerem que, em qualquer situação, as funções $W=W(t)$ e $Q=Q(t)$ não sejam deriváveis, para todos os valores da variável independente. Este fato não poderia ser representado por uma função como $x^{2}$, ou como $\tan x$, usadas nos parágrafos anteriores, porque para elas a derivada não existe, no máximo, em pontos isolados. Além disto, este fato seria uma característica intrínseca do trabalho e do calor, a qual não poderia ser alterada ao se passar de uma teoria para outra (assim como o fato do tempo não retroceder é característica intrínseca do tempo, que não pode ser alterada ao se passar de uma teoria para outra). Mas, se forem verificadas as formas das funções $W=W(t)$ e $Q=Q(t)$ em eletrodinâmica, mecânica e transmissão condutiva de calor, ver-se-á que estas funções são deriváveis em um ou mais pontos. Portanto, em qualquer teoria a inexistência destas derivadas pode ocorrer, mas não em qualquer situação, para todos os valores da variável independente.

O mais provável, porém, não é que Anacleto e Anacleto ${ }^{35}$ considerem que as funções não sejam deriváveis, mas sim que considerem que tais funções simplesmente não existam. Entretanto, isto seria impossível, não apenas porque se trataria de uma característica intrínseca (as funções existem em outras teorias, conforme já afirmado), mas também porque, a cada instante durante a realização de um processo termodinâmico, corresponde um e apenas um trabalho realizado até aquele momento, bem como um e apenas um calor trocado até aquele momento (esta é, exatamente, a definição matemática de função e, certamente, a definição matemática de função não varia de acordo com a teoria física considerada). Portanto, mesmo considerando exclusivamente a termodinâmica, ou seja, mesmo sem fazer comparações com outras teorias, tais funções necessariamente existem.

Ressalte-se, ainda, que as características intrínsecas que tornam algumas grandezas descritíveis (em toda e qualquer teoria) por meio de equações diferenciais exatas (funções de estado), enquanto que outras são descritas por equações diferenciais inexatas (funções de processo), nada têm a ver com a existência, ou não, de derivadas temporais. Este tópico será tratado na próxima seção, mas, desde 
já, pode ser adiantado que as mencionadas equações diferenciais exatas e inexatas envolvem derivadas em relação a propriedades que caracterizam o estado do sistema, absolutamente não envolvendo derivadas temporais. Tanto é assim que um dos conceitos centrais da termodinâmica atemporal é a distinção entre função de estado e função de processo, distinção esta também presente na termodinâmica temporal.

Em resumo, o equívoco na argumentação de Anacleto e Anacleto ${ }^{35}$ está em afirmar que calor e trabalho não são deriváveis temporalmente, ao invés de simplesmente reconhecer que a termodinâmica tradicional, bem como a termodinâmica de Gibbs, não utiliza tais derivadas. Gibbs jamais afirmou que as derivadas temporais do calor e do trabalho não existam. Aliás, até mesmo os livros didáticos de termodinâmica tradicional não costumam fazer tal afirmação. Para defender a atemporalidade, convém ater-se a Gibbs (que, evidentemente, incluiu a atemporalidade em sua teoria não por considerar que a natureza assim o seja, mas por priorizar a perfeita coerência matemática), porque é impossível contestar a solidez conceitual da termodinâmica atemporal de Gibbs. Por outro lado, é temerário defender a atemporalidade por meio de elaborações baseadas na termodinâmica tradicional, devido à forte probabilidade de, sem perceber, escorregar para ilogismos.

\section{FUNÇÃO DE ESTADO E DE PROCESSO}

Seja $z=z(x, y)$ uma função contínua e diferenciável, cuja equação diferencial $d z=A d x+B d y$, sendo $A=\frac{\partial z(x, y)}{\partial x}$ e $B=\frac{\partial z(x, y)}{\partial y}$, define a relação entre os diferenciais $d z, d x$ e $d y$. Lembrando que a equação diferencial existe sempre que exista a função, mas que o inverso não é verdadeiro (neste último caso, evidentemente, não existem as igualdades $A=\frac{\partial z(x, y)}{\partial x}$ e $\left.B=\frac{\partial z(x, y)}{\partial y}\right)$, há dois aspectos a serem destacados:

1. Se a função $z=z(x, y)$ existir, a equação será uma equação diferencial exata. Nesse caso, o conjunto de valores $\langle\mathrm{x}, \mathrm{y}\rangle$, correspondente ao instante $t$, definirá completamente um único valor de $z$. Tal valor, portanto, independerá dos valores históricos de x e y, anteriores ao instante $t$. Quando $z=z(x, y)$ existir, se $x$, y e $z$ forem os valores de três diferentes propriedades do sistema no instante $t$ e o conjunto de valores $\langle x, y\rangle$ for suficiente para definir o estado do sistema, $z$ será denominada função de estado.

2. Se a função $z=z(x, y)$ não existir, a equação será uma equação diferencial inexata. Nesse caso, o valor de $z$ correspondente a cada conjunto de valores $\langle x, y\rangle$ poderá depender dos valores históricos de $x$ e $y$, anteriores ao instante considerado. Quando esta dependência ocorrer, se $z$ for o valor de uma grandeza referente ao processo, como o calor ou o trabalho nele envolvidos, $z$ será denominada função de processo.

Sugere-se que a definição matemática correta de diferencial seja procurada em um bom livro de cálculo como, por exemplo, Apostol, ${ }^{36}$ porque definições simplistas, infelizmente também encontráveis em livros de cálculo matemático, podem ser extremamente prejudiciais à compreensão da termodinâmica. ${ }^{31}$ Deve-se alertar que, ao contrário do que afirma a termodinâmica tradicional e somente ela, não existem diferenciais exatos e inexatos, ${ }^{1,2,32}$ mas apenas equações diferenciais exatas e inexatas. Em relação a esta última afirmação, sugere-se também consultar um bom livro de equações diferenciais como, por exemplo, Ince. ${ }^{37}$ Para o leitor que deseje pesquisar profundamente o tema, propõe-se a leitura do trabalho original do grande matemático Dedekind, ${ }^{38}$ que estabeleceu o conceito moderno de diferencial. Para uma leitura complementar ao trabalho de Dedekind, sugere-se Cantor. ${ }^{39}$
Vale ressaltar que tanto uma equação diferencial exata, como uma inexata, é uma equação diferencial definidora da relação entre os três diferenciais $d z, d x$ e $d y$. Mas a integração ordinária, para produzir a função primitiva $z=z(x, y)$, só é possível no caso da equação diferencial exata. No caso da inexata, a integração somente pode ser efetuada mediante o fornecimento de informação adicional ao conhecimento da equação diferencial $d z=A d x+B d y$. Por exemplo, para uma função de processo uma integral de linha pode ser calculada, mediante o fornecimento de suficiente informação sobre o processo em questão.

\section{PRIMEIRA LEI DA TERMODINÂMICA}

O conteúdo energético total de um sistema pode ser descrito por $E=E_{C R}+U$, sendo $E_{C R}$ a energia de corpo rígido (a energia que não envolve alterações nas distâncias, ou nos ângulos entre as partículas, mas sim no momento linear, ou angular, ou ambos, do sistema como um todo) e sendo $U$ a energia interna (a energia que envolve alterações nas distâncias, ou nos ângulos entre as partículas, ou ambos, mas não no momento linear, ou no momento angular, do sistema como um todo). Para um sistema isolado (sistema que não troca nem massa, nem energia com o seu exterior), a energia de corpo rígido não pode ser convertida em energia interna ou vice-versa. ${ }^{9,40}$ Portanto, para um sistema fechado (sistema que não troca massa com o seu exterior) tem-se $\Delta E=\Delta E_{C R}+\Delta U$, sendo cada uma das alterações $\Delta E_{C R}$ e $\Delta U$ exclusivamente devida a trocas entre o sistema e o seu exterior.

O calor $\Delta Q$ é uma transmissão de energia que mantém inalterado o momento linear total e o momento angular total de cada um dos sistemas que trocam energia entre si. Logo, as energias de corpo rígido destes sistemas não podem ser modificadas por meio de transmissão de calor, que somente pode ser absorvido ou emitido por energias internas. A outra possibilidade de transmissão de energia não está sujeita à restrição de não alterar os momentos lineares totais, ou os momentos angulares totais dos sistemas em contato energético. Ela é chamada trabalho. Este, pode ser trocado tanto pelas energias internas, sendo então grafado $\Delta W$, como pelas energias de corpo rígido, quando é simbolizado por $\Delta W_{C R}$. Logo, a denominação trabalho inclui qualquer tipo de transmissão de energia entre sistema e exterior, salvo calor.

Portanto, para um sistema fechado tem-se $\Delta U=\Delta Q+\Delta W$ e $\Delta E_{C R}$ $=\Delta W_{C R}$. Somando-se as duas igualdades e lembrando que $\Delta E=\Delta E_{C R}$ $+\Delta U$, obtém-se $\Delta E=\Delta Q+\Delta W_{C R}+\Delta W$, ou $\Delta E=\Delta Q+\Delta W_{T O T}$, sendo esta última a equação global de balanceamento de energia para um sistema fechado. Note que, de início, propositalmente utilizou-se o mesmo símbolo $\Delta$ tanto para as alterações energéticas do sistema $\Delta E, \Delta E_{C R}$ e $\Delta U$, como para as trocas com o exterior $\Delta Q, \Delta W, \Delta W_{C R}$ e $\Delta W_{T O T}$. Note, também, que a equação de balanceamento $\Delta E=\Delta Q$ $+\Delta W_{T O T}$ contém menos informação que o conjunto de equações em que ela pode ser decomposta, $\Delta E=\Delta E_{C R}+\Delta U, \Delta W_{T O T}=\Delta W_{C R}+\Delta W$, $\Delta E_{C R}=\Delta W_{C R}$ e $\Delta U=\Delta Q+\Delta W$. A termodinâmica focaliza somente a última destas equações, porque deixa para a mecânica o estudo da energia de corpo rígido.

Durante o tempo de existência de um processo homogêneo, o valor de cada uma das propriedades intensivas do sistema pode variar no tempo, mas em qualquer instante tal valor é bem definido para o sistema como um todo, porque é o mesmo em todos os pontos geométricos do sistema. Suponha que o estado de um sistema fechado constituído por uma única substância pura, a qual sofra um processo homogêneo, possa ser caracterizado pelos valores da entropia e do volume do sistema. Neste caso, a energia interna $U$ é uma função de estado, ou seja, ela é uma função da entropia e do volume do sistema, o que pode ser escrito $U=U(S, V)$.

De fato, o valor de $U$ pode ser medido em cada estado represen- 
tado por algum possível conjunto de valores $\langle S, V\rangle$, relativamente a um estado referencial arbitrariamente fixado e as medidas mostram que, a cada conjunto $\langle S, V\rangle$, corresponde um e apenas um valor $U$. O conhecimento do valor $U$, portanto, não exige que sejam efetuadas medidas em outro instante que não aquele em que ocorre o estado representado por $\langle S, V\rangle$, nem exige informações sobre o processo no qual este estado se insira. A função $U=U(S, V)$ é diferenciável para todo conjunto de valores $\langle S, V\rangle$ pertencente ao processo. Logo, a equação diferencial definidora da relação entre $d U, d S$ e $d V$ é uma equação diferencial exata.

A homogeneidade do processo garante que, em cada instante $t$ pertencente ao tempo de existência do processo, as derivadas parciais de $U$ em relação a $S$ e $V$, derivadas estas que são propriedades intensivas do sistema, apresentem valores respectivamente iguais em todos os pontos geométricos do sistema. Denominando as derivadas parciais de $U=U(S, V)$, em relação a $S$ e $V$, respectivamente $T$ e $-P$, tem-se $d U=T d S-P d V$. Este assunto será retomado na seção Equação TdS.

Mas a energia interna, também, pode ser descrita como função do instante $t$ no qual ocorre o estado caracterizado por $S$ e $V$, instante este pertencente ao tempo de existência do processo, $\log o U=U(t)$. Esta função existe, pois para cada valor de $t$ há um único valor de $U$ e, também, valores únicos respectivamente para $S$ e $V$, portanto há um único estado. Considere, também, a definição $\dot{U}=\frac{d U}{d t}$, logo considere que a função $U=U(t)$ seja diferenciável e que a equação diferencial definidora da relação entre $d U$ e $d t$ seja $d U=\dot{U}(t) d t$.

Vale ressaltar que tanto os valores da função $U=U(t)$, quanto os da função $\dot{U}=\dot{U}(t)$, só dependem do instante $t$, independendo portanto do histórico do processo. Porém, $\dot{U}$ não é uma função de estado, portanto não existe uma função que, dado algum possível conjunto de valores $\langle S, V\rangle$, forneça o valor de $\dot{U}$. Aliás, toda derivada temporal de uma propriedade, ou função de estado, não é uma função de estado. Isto ocorre precisamente porque o valor de qualquer propriedade, em qualquer momento, é totalmente independente do valor da sua derivada temporal, no mesmo instante. De fato, lançando a propriedade e o tempo em dois eixos ortogonais, um ponto, que no gráfico resultante representa o valor da propriedade considerada em determinado instante, pode pertencer a infinitas curvas que por ele passem (a derivada é o valor do coeficiente angular da reta tangente à curva, no ponto considerado).

Para melhor entender porque $U$ é função de estado, mas $\dot{U}$ não é, suponha que os valores de $U, S$ e $V$ sejam lançados em três eixos ortogonais, a cada conjunto $\langle S, V\rangle$ correspondendo um valor $U$. Neste caso, a função $U=U(S, V)$ descreve uma superfície no espaço tridimensional considerado e a equação diferencial definidora da relação entre $d U, d S$ e $d V$, grafada $d U=T d S-P d V$, informa qual será a tendência de variação de $U$ em determinado ponto da superfície $U=U(S, V)$, dadas as tendências de variação de $S$ e $V$ naquele ponto. O ponto, neste caso, não é um ponto geométrico do sistema, mas sim um determinado conjunto $\langle S, V\rangle$, que define os valores $T=$ $T(S, V)$ e $P=P(S, V)$.

Mas a equação diferencial definidora da relação entre $d U, d S$ e $d V$ não informa quais, efetivamente, serão os valores numéricos das três tendências naquele ponto $\langle S, V\rangle$, que ocorre no instante $t$, grafadas $\dot{U}=\dot{U}(t), \dot{S}=\dot{S}(t)$ e $\dot{V}=\dot{V}(t)$. De fato, $d U=T d S-P d V$ corresponde a $\dot{U}=T \dot{S}-P \dot{V}$, porque $d U=\dot{U} d t, d S=\dot{S} d t$ e $d V=\dot{V} d t$. Porém, $d U=T d S-P d V$ representa um plano onde $d S$ e $d V$ são as variáveis independentes e $d U$ é a variável dependente, sendo $T=$ $T(S, V)$ e $P=P(S, V)$ dois números bem definidos no ponto $\langle S, V\rangle .^{36}$ O conhecimento de qual é o ponto $\langle S, V>$ é suficiente para determinar o citado plano e, também, a relação $\dot{U}=T \dot{S}-P \dot{V}$, mas é insuficiente para determinar os três valores numéricos $\dot{U}=\dot{U}(t), \dot{S}=\dot{S}(t)$ e $\dot{V}=$
$\dot{V}(t)$, que ocorrem no instante $t$, porque $t$ determina $\langle S, V\rangle$, mas $\langle S, V\rangle$ não determina $t .^{1}$

Para perceber isto, considere o vetor $\vec{X}=\langle S, V\rangle$. Como $t$ determina $\langle S, V\rangle$, tem-se o processo $\vec{X}=\vec{X}(t)$. Para que $\langle S, V\rangle$ determinasse $t$, seria necessário que existisse a função inversa, $t=t(\vec{X})$, a qual indicaria que a todo vetor $\vec{X}$, dentro de um domínio onde as propriedades incluídas em $\vec{X}$ variam continuamente, corresponderia um valor $t$. Lançando os valores $t, S$ e $V$ em três eixos ortogonais, $\vec{X}$ $=\vec{X}(t)$ representa uma curva [a curva definida por $S=S(t)$ e $V=V(t)$ ], enquanto que $t=t(\vec{X})$ representa uma superfície [a superfície definida por $t=t(S, V)]$. Lembrando que uma superfície é formada por infinitas curvas, percebe-se que é impossível inverter $\vec{X}=\vec{X}(t)$.

É claro que é possível restringir-se aos pontos desta superfície cujo argumento $\vec{X}$ sejam os pontos da curva $\vec{X}=\vec{X}(t)$, o que significa escolher uma curva específica na superfície. Esta específica curva, se nenhum estado englobado por $\vec{X}=\vec{X}(t)$ se repetir, sem dúvida será uma função $t=t(\vec{X})$. Portanto, dado um bem definido processo $\vec{X}=$ $\vec{X}(t)$, pode existir a correspondente função $t=t(\vec{X})$. Logo, para um processo bem definido, pode-se determinar os três valores numéricos $\dot{U}=\dot{U}(t), \dot{S}=\dot{S}(t)$ e $\dot{V}=\dot{V}(t)$, que ocorrem no instante $t=t(\vec{X})$. Mas isto não indica que $\dot{U}, \dot{S}$ e $\dot{V}$ sejam funções de estado, precisamente porque, para se chegar nestas determinações, exige-se informação sobre o processo ocorrido.

Assim como existe a função $U=U(t)$, também existe a função $Q$ $=Q(t)$, conforme já colocado. Sendo $t_{0} \mathrm{o}$ instante inicial do processo e impondo-se $Q\left(t_{0}\right)=0$, a função $Q=Q(t)$ será tal que, a cada instante $t$, corresponda um valor para $Q$ igual ao calor trocado desde o início do processo até ao instante $t$. Portanto, conforme também já foi afirmado, o calor é uma função de processo. Considere a potência calorífica $\frac{d Q}{d t}=\dot{Q}, \log$ imponha que a função $Q=Q(t)$ seja diferenciável e que a equação diferencial definidora da relação entre $d Q$ e $d t$ seja $d Q=\dot{Q} d t$. A potência $\dot{Q}=\dot{Q}(t)$ também é uma função do tempo, mas o seu valor só depende do instante considerado, independendo portanto do que aconteceu em momentos anteriores. Por exemplo, para dois sistemas em contato diatérmico a temperaturas diferentes $T_{1}$ e $T_{2}$, o valor de $\dot{Q}$ depende apenas da diferença de temperatura e das características dos dois sistemas no instante considerado, não dependendo do histórico do processo (lei de Fourier para a transmissão condutiva de calor).

Mas o fato do valor de $\dot{Q}$ depender da temperatura exterior evidencia que não se trata de uma função de estado, a qual depende apenas dos valores das propriedades que caracterizam o estado do sistema. A mesma discussão é valida para o trabalho $W$ e, portanto, tem-se $W$ $=W(t), \dot{W}=\dot{W}(t)$ e $d W=\dot{W} d t$. Pode-se escrever a primeira lei como sendo $\dot{U}=\dot{Q}+\dot{W}$ e pode-se, também, escrever $d U=d Q+d W$, porque tem-se $d U=\dot{U} d t, d Q=\dot{Q} d t$ e $d W=\dot{W} d t$. A interpretação de $U=U(t)$ pode ser análoga à de $Q=Q(t)$ e $W=W(t)$. De fato, a função $U=U(t)$ pode ser tal que a cada instante $t$ corresponda um valor de $U$ igual à variação de energia interna do sistema, desde o início do processo até ao instante $\mathrm{t}$, bastando para isto impor que $U(t)=\int_{t_{0}}^{t} \dot{U}(t) d t$, sendo $U\left(t_{0}\right)=0$. A equação $U\left(t_{0}\right)=0$ pode ser arbitrariamente imposta, ou não, dependendo da conveniência, porque apenas variações de energia interna são mensuráveis, não porque a energia interna do sistema, no instante inicial, de verdade seja nula.

Aliás, $Q\left(t_{0}\right)$ e $W\left(t_{0}\right)$ são respectivamente o calor e o trabalho trocados entre o sistema e seu exterior, até ao instante inicial $t_{0}$ e desde um momento referencial do sistema, momento este anterior ou igual a $t_{0}$. Como tal momento referencial é arbitrário, é possível fazê-lo coincidir com $t_{0}$, ou seja, convencionar $Q\left(t_{0}\right)=0$ e $W\left(t_{0}\right)=0$ é permitido e, além disto, é muito conveniente. No que se refere à energia interna, como ela é conhecida a menos de uma constante aditiva, fazer $U\left(t_{0}\right)=0$ também é permitido. Mas pode ser mais conveniente adotar 
outra convenção, como considerar que a energia interna do sistema seja nula num bem determinado estado referencial (por exemplo, no zero absoluto). Convenções relativas a algum estado referencial do sistema, evidentemente só são possíveis para funções de estado, como é o caso da energia interna, não sendo possíveis para funções de processo, como o calor e o trabalho.

Em resumo, a equação $d U=d Q+d W$, tendo seus diferenciais definidos por $d U=\dot{U} d t, d Q=\dot{Q} d t$ e $d W=\dot{W} d t$, logo implicando em $\dot{U}=\dot{Q}+\dot{W}$, ao ser integrada entre o instante inicial $t_{0}$ e o momento genérico $t$ pertencente ao tempo de existência do processo, evidentemente produz $\Delta U=\Delta Q+\Delta W$, equação esta que aparece no terceiro parágrafo desta seção. Existe, portanto, total coerência matemática entre a equação para o processo finito que ocorre desde o instante $t_{0}$ até ao momento $t$, grafada $\Delta U=\Delta Q+\Delta W$ e as equações $\dot{U}=\dot{Q}+\dot{W}$ e $d U=d Q+d W$, referentes a qualquer instante pertencente ao tempo de existência do processo. ${ }^{1,2}$ Como $\Delta U=U(t)-U\left(t_{0}\right), \Delta Q=Q(t)-$ $Q\left(t_{0}\right), \quad \Delta W=W(t)-W\left(t_{0}\right)$ e, geralmente, convenciona-se $Q\left(t_{0}\right)=0$, $W\left(t_{0}\right)=0$, mas $U\left(t_{0}\right) \neq 0$, chega-se à expressão $\Delta U=Q+W$, apresentada no primeiro parágrafo da Introdução.

Para Anacleto e Anacleto, ${ }^{35}$ a desigualdade $d(Q+W) / d t \neq(d Q / d t)$ $+(d W / d t)$ poderia ocorrer. Como a derivada da soma é a soma das derivadas, isto implicaria em que a troca energética entre o sistema e o seu exterior não fosse separável nas parcelas calor e trabalho. Em outras palavras, implicaria em que as transmissões de energia entre sistemas não fossem separáveis numa parcela que não altera os momentos lineares totais e angulares totais dos sistemas em contato energético e numa outra, para a qual não há esta restrição. Isto só ocorreria se não existisse, na natureza, um tipo de transmissão de energia sujeito à mencionada restrição.

\section{PROCESSO TEMPORAL E PROCESSO REVERSÍVEL}

Seja $\vec{X}=\vec{X}(t)$ um vetor que caracterize o estado que ocorre no instante $t$, por exemplo, $\vec{X}(t)=<S(t), V(t)>$. Considere o processo $\vec{X}$ $=\vec{X}(t)$, com estados inicial e final, respectivamente, $\vec{X}=\vec{A}$ e $\vec{X}=$ $\vec{B}$. Este processo corresponde a um segmento de curva no espaço tridimensional definido pelos eixos ortogonais onde são lançados os valores de $S, V$ e $t$. O processo reverso apresenta os mesmos estados do processo direto, mas não o mesmo segmento de curva. No processo reverso, os estados do sistema são repetidos na ordem cronológica oposta à que ocorreu no processo direto, mas o tempo evidentemente continua avançando. Supondo que um espelho plano perpendicular ao eixo no qual são lançados os valores de $t$ passe por $\vec{X}=\vec{B}$, o segmento de curva correspondente ao processo reverso é a imagem especular daquele referente ao processo direto.

Se $\vec{A}=\vec{X}\left(t_{A}\right), \vec{B}=\vec{X}\left(t_{B}\right)$ e $t_{A} \leq t \leq t_{B}$, a imagem especular de $\vec{X}$ $=\vec{X}(t)$ ocorrerá no instante $t_{B}+\left[\left(t_{B}-t_{A}\right)-\left(t-t_{A}\right)\right]=2 t_{B}-t$. Logo, a definição matemática de processo reverso é $\vec{X}\left(2 t_{B}-t\right)=\vec{X}(t)$. Este é o conceito de processo reverso, conforme Boltzmann. ${ }^{14}$ Num processo reversível, o processo reverso apresenta, para cada estado $\vec{X}$, potências $\dot{W}$ e $\dot{Q}$ com módulos iguais, mas sinais opostos às potências respectivamente apresentadas pelo mesmo estado $\vec{X}$ do processo direto. Portanto, num processo reversível $W\left(2 t_{B}-t\right)=-W(t)$ e $Q\left(2 t_{B}\right.$ $-t)=-Q(t)$, para $\vec{X}\left(2 t_{B}-t\right)=\vec{X}(t)$.

Seja $E=E(t)$ uma propriedade ou função de estado extensiva qualquer. Ser contínua e diferenciável a função $E=E(t)$, não implica em que ela seja invertível. ${ }^{36}$ De fato, num processo qualquer, para cada valor de $t$ há um único valor de $E$, porém para cada valor de $E$ não há necessariamente um único valor de $t$, mesmo que se tratasse de um processo no qual nenhum estado se repetisse, ou seja, no qual cada vetor $\vec{X}$ correspondesse a um único instante $t$. Por exemplo, mesmo que nenhum estado se repetisse, nada garantiria que dois ou mais estados com mesmo volume (ou com a mesma entropia, ou com a mesma energia interna etc.) não fossem contidos pelo mesmo processo. Logo, não existe $t=t(E)$. Para uma função de estado intensiva, escrever $I=I(t)$ ou implica em processo homogêneo, ou em que exista um valor $I=I(t)$ específico para cada ponto do sistema. Em ambos os casos, a função $t=t(I)$ não existe, por razões análogas às apresentadas para a função $E=E(t)$.

Não é claro se Anacleto e Anacleto ${ }^{35}$ consideram que o colocado por Moreira e Bassi ${ }^{27}$ implica na existência das funções $t=t(E)$ e $t$ $=t(I)$, mas certamente eles acham que implica na existência da função $t=t(\vec{X})$. Porém, não implica e tal função não existe, conforme mostrado na seção Primeira Lei da Termodinâmica, para $\vec{X}=\langle S, V\rangle$. Eles, também, afirmam que "admitir que existem as funções diferenciáveis $Q=Q(t)$ e $W=W(t)$ pode significar que todos os processos são reversíveis (no tempo), o que é falso." $\mathrm{O}$ fato das funções $Q=$ $Q(t)$ e $W=W(t)$ serem diferenciáveis apenas informa que elas são contínuas e que não apresentam ângulos, não que elas sejam invertíveis, por razões análogas às apresentadas para $E=E(t)$ e $I=I(t)$. Por exemplo, um processo pode apresentar o mesmo valor $Q=Q(t)$ em diversos instantes $t$. Mas, mesmo impondo-se que o processo jamais repita o valor $Q$, ou seja, mesmo nos casos particulares onde exista $t=t(Q)$, isto não significa que $Q\left(2 t_{B}-t\right)=-Q(t)$, para $\vec{X}\left(2 t_{B}-t\right)=$ $\vec{X}(t)$. Analogamente para $W=W(t)$.

\section{EQUAÇÃO TdS}

De acordo com a termodinâmica tradicional, a equação $d U=T d S$ - $P d V$, que será chamada equação TdS, é deduzida para o caso em que o único trabalho trocado com o exterior é volumétrico expansivo, para processos reversíveis em sistemas fechados sem reação química. Neste caso, esta equação é obtida a partir da forma diferencial da primeira lei da termodinâmica $d U=d Q+d W$, porque $d Q=T d S$ e $d W=-P d V$. Anacleto e Anacleto, ${ }^{35}$ porém, consideram que, como apenas funções de estado estão envolvidas na igualdade $d U=T d S-$ $P d V$, ela continua válida mesmo que o processo não seja reversível. Este é um grave erro matemático, frequentemente encontrado em livros didáticos de termodinâmica tradicional. De fato, se fossem finitas as diferenças, o argumento seria absolutamente correto. $\mathrm{Ou}$ seja, se os estados inicial e final de um processo estivessem ambos em equilíbrio termodinâmico, respectivamente nas mesmas temperatura $T$ e pressão $P$, caso a igualdade $\Delta U=T \Delta S-P \Delta V$ valesse para um processo reversível interligando tais estados, também valeria quando um processo irreversível interligasse os mesmos estados, exatamente porque apenas funções destes dois estados terminais se encontram envolvidas nesta igualdade. Mas um diferencial não é um pequeníssimo intervalo finito.

As idéias de infinitésimo e de diferencial, ${ }^{31,32,36-39}$ por envolverem o conceito matemático de limite, referem-se a algo pontual, não a um pequeníssimo intervalo finito. Como tanto $\dot{U}=\dot{Q}+\dot{W}$, quanto $d U=$ $d Q+d W$, referem-se a um só instante e a um só estado, tal estado não pode ser simultaneamente de equilíbrio e de não equilíbrio. Se as igualdades $d Q=T d S$ e $d W=-P d V$ forem válidas apenas num estado de equilíbrio, então $d U=d Q+d W$ não implica em $d U=$ $T d S-P d V$, para um estado de não equilíbrio. Tratar um diferencial como um pequeníssimo intervalo finito é um dos erros mais comuns da termodinâmica tradicional., ${ }^{1,2,31,32}$

Entretanto, para a equação TdS existe uma interpretação dada pela termodinâmica dos processos homogêneos, interpretação esta já mencionada na seção Primeira Lei da Termodinâmica. Conforme tal interpretação, sempre que o estado do sistema possa ser definido pelo vetor $\vec{X}(t)=\langle S(t), V(t)>$, como no caso de um sistema fechado constituído por uma única substância pura, a qual sofra um processo homogêneo, denominando as derivadas parciais de $U=U(S, V)$, em relação a $S$ e $V$, respectivamente $T$ e $-P$, tem-se $d U=T d S-P d V$. Note 
que esta outra interpretação, em relação à primeira, retira a exigência de reversibilidade, logo retira a exigência de equilíbrio termodinâmico. Por outro lado, explicita a exigência de homogeneidade termobárica para o estado considerado (embora tanto a temperatura quanto a pressão possam variar com o tempo), o que é esquecido pelo enfoque tradicional, quando a reversibilidade é retirada (um estado qualquer, pertencente a um processo irreversível, não é termobaricamente homogêneo).

Seja um determinado volume interior ao sistema considerado (o qual foi suposto conter uma substância pura), separado do restante por uma fronteira imaginária. Tal subsistema aberto, ou volume de controle conforme frequentemente denominado em engenharia química, será regido pela equação $d U=T d S-P d V+\mu d N$, de acordo com a termodinâmica dos processos homogêneos, onde $\mu=\frac{\partial U(S, V, N)}{\partial N}$ é o potencial químico da substância pura em questão. Isto acontece porque, no subsistema aberto, $U=U(S, V, N)$, sendo $N$ a quantidade de matéria do subsistema no instante $t$. Em toda ciência natural clássica, o comportamento macroscópico da matéria pode ser extrapolado para regiões tão pequenas quanto se queira, para permitir a aplicação do cálculo diferencial e integral às leis macroscópicas que regem a ciência considerada. Por isto, pode-se fazer o subsistema tender para um ponto.

Entretanto, qualquer propriedade extensiva tenderá para zero quando o subsistema tender para um ponto, o que leva à substituição de $U=U(S, V, N)$, por exemplo, por $u=u(s, v, n)$, onde $u=$ $U / M, s=S / M, v=V / M$ e $n=N / M$, sendo $M$ a massa do subsistema no instante $t$. Tem-se então, ao invés de $d U=T d S-P d V+\mu d N$, a equação diferencial $d u=T d s-P d v+\mu d n$, válida também quando as dimensões do subsistema tenderem para um ponto, porque nenhuma das propriedades envolvidas nesta última equação é necessariamente nula, em qualquer ponto geométrico do sistema.

Porém, todo subsistema de um sistema heterogêneo contínuo tenderá à homogeneidade quando o volume do subsistema tender para zero. Logo, a equação $d u=T d s-P d v+\mu d n$ será válida em todos os pontos geométricos de qualquer sistema fechado contínuo contendo uma única substância pura, variando de ponto para ponto os valores das propriedades intensivas $T, P$ e $\mu$, em cada instante $t$. Se houver mais do que uma substância, bastará usar $d u=T d s-P d v+\sum_{j}^{J} \mu_{j} d n_{j}$, onde $J$ é o número de substâncias presentes. Esta última é uma das equações fundamentais em que se baseia toda a termodinâmica do não equilíbrio, bem como a termodinâmica dos meios contínuos.

Cabe ainda lembrar que o sistema pode trocar com o exterior outros tipos de trabalho que não apenas o volumétrico, os quais evidentemente deverão ser incluídos na expressão da primeira lei, seja o processo reversível, ou não. Mas a expressão $d u=T d s-P d v+\sum_{j}^{J} \mu_{j} d n_{j}$, ou $d U=T d S-P d V+\sum_{j}^{J} \mu_{j} d N_{j}$ para o caso de processo homogêneo, pode permanecer inalterada. Exemplo típico é o funcionamento de uma célula eletroquímica. A carga elétrica da célula como um todo não se altera durante o seu funcionamento, o que significa que, embora ocorra um trabalho elétrico, na aproximação homogênea tem-se $U=U\left(S, V, N_{1}, N_{2}, \ldots N_{J}\right)$, porque a carga elétrica $Q$ do sistema é uma constante, logo $d U=T d S-P d V+\sum_{j}^{J} \mu_{j} d N_{j}$.

Por outro lado, se um sistema se expandir contra uma pressão externa nula não haverá realização de trabalho volumétrico, portanto este termo não aparecerá na expressão da primeira lei, embora o volume se altere, logo, precise ser mantido como propriedade que define o estado do sistema. A equação TdS e a forma diferencial da primeira lei, portanto, coincidem apenas em casos muito particulares. Como expressões gerais, não se pode deduzir uma a partir da outra. A equação TdS existe porque existem funções de estado deriváveis, enquanto que a primeira lei existe porque a energia se conserva e porque, num sistema isolado, a energia interna e a energia de corpo rígido não se transformam uma na outra. A forma diferencial da primeira lei existe exclusivamente porque $Q$ e $W$ são deriváveis no tempo e, além disto, o mesmo acontece com qualquer propriedade ou função de estado, logo acontece com $U$.

\section{CONCLUSÃO}

Este trabalho não pretende ser, apenas, uma contra-argumentação ao artigo de Anacleto e Anacleto, ${ }^{35}$ porque isto muito o empobreceria. $\mathrm{O}$ artigo de Moreira e Bassi, ${ }^{27}$ que eles contestaram 6 anos após sua publicação e que nunca antes foi criticado, somente discute, sob o ponto de vista da termodinâmica dos processos homogêneos, a primeira lei da termodinâmica. Precisamente porque o artigo de Moreira e Bassi $^{27}$ nem sequer se aprofunda em descrever a termodinâmica dos processos homogêneos, ele pode dar a impressão de não estar baseado em fundamentos sólidos. Fez-se, portanto, necessário mostrar, nesta contra-argumentação, todo o arcabouço teórico no qual ele se fundamenta.

\section{REFERÊNCIAS}

1. Truesdell, C. A.; Rational Thermodynamics, Springer: New York, 1984.

2. Truesdell, C. A.; Bharatha, S.; The Concepts and Logic of Classical Thermodynamics as a Theory of Heat Engines - Rigorously Constructed upon the Foundations Laid by S. Carnot and F. Reech, Springer: New York, 1988.

3. Truesdell, C. A.; Toupin, R.; Handbuch der Physik, Springer: Berlin, 1960.

4. Day, W. A.; The Thermodynamics of Simple Materials with Fading Memory, Springer: New York, 1972.

5. Serrin, J. Em International Symposium on Continuum Mechanics and Partial Differential Equations at Rio de Janeiro; de La Penha, G. M.; Medeiros, L. A., eds.; North-Holland: Amsterdam, 1978.

6. Owen, D. R.; A First Course in the Mathematical Foundations of Thermodynamics, Springer: New York, 1984.

7. Silhavý, M.; The Mechanics and Thermodynamics of Continuous Media, Springer: New York, 1997.

8. Muller, I.; Ruggeri T.; Rational Extended Thermodynamics, Springer: New York, 1998.

9. Serrin, J. Em Nonlinear Analysis and Continuum Mechanics; Buttazzo G.; Galdi G. P.; Lanconelli E.; Pucci P., eds.; Springer: New York, 1998.

10. Liu, I-S.; Continuum Mechanics, Springer: Berlin, 2002.

11. Carnot, S.; Réflexions sur la Puissance Motrice du Feu et sur les Machines propres à Développer cette Puissance, Bachelier: Paris, 1824.

12. Truesdell, C. A.; The Tragicomical History of Thermodynamics: 1822-1854, Springer: New York, 1980.

13. Gibbs, J. W.; Elementary Principles in Statistical Mechanics developed with especial reference to the rational foundations of thermodynamics, Yale: New York, 1902.

14. Tolman, R. C.; The Principles of Statistical Mechanics, Oxford: New York, 1938.

15. Hill, T. L.; An Introduction to Statistical Thermodynamics, Addison Wesley: Reading, 1960.

16. Planck, M. K. E. L.; Vorlesungen über Thermodynamik, Veit: Leipzig, 1891.

17. Planck, M. K. E. L.; Wissenschaftliche Selbstbiographie, Barth: Leipzig, 1948.

18. Prigogine I.; Defay, R.; Chemical Thermodynamics, Longmans, Green: London, 1954.

19. Prigogine, I.; Nonequilibrium Statistical Mechanics, Interscience: New York, 1960.

20. De Groot, S. R.; Mazur, P.; Non-equilibrium Thermodynamics, NorthHolland: Amsterdam, 1962. 
21. Onsager, L.; Phys. Rev. A 1931, 37, 405.

22. Onsager, L.; Phys. Rev. A 1931, 38, 2265.

23. Eckart, C.; Phys. Rev. A 1940, 58, 267.

24. Casimir, H. B. G.; Review of Modern Physics 1945, 17, 343.

25. Miller, D. G.; Chem. Rev. 1960, 60, 15.

26. Kondepudi, D.; Prigogine, I.; Modern Thermodynamics-From Heat Engines to Dissipative Structures, Wiley: New York, 1998.

27. Moreira, N. H.; Bassi, A. B. M. S.; Quim. Nova 2001, 24, 563.

28. Nery, A. R. L.; Dissertação de Mestrado, Universidade Estadual de Campinas, Brasil, 2007.

29. The Second Law of Thermodynamics; Kestin, J., ed.; Dowden, Hutchinson and Ross: Stroudsburg, 1976.

30. Lewis, G. N.; Randall, M.; Thermodynamics, McGraw-Hill: New York, 1961.

31. http://www.chemkeys.com/artigo/41/0, acessada em Janeiro de 2009.
32. http://www.chemkeys.com/artigo/47/0, acessada em Janeiro de 2009.

33. Debenedetti, P. G.; Metastable Liquids, Princeton: Princeton, 1996.

34. Liquids Under Negative Pressure (NATO Science Series); Imre, A. R.; Maris, H. J.; Williams, P. R., eds.; Kluwer: Dordrecht, 2002.

35. Anacleto, J.; Anacleto, A.; Quim. Nova 2007, 30, 488.

36. Apostol, T. M.; Calculus, Wiley: New York, 1967, vol. 1.

37. Ince, E. L.; Ordinary Differential Equations, Longmans, Green: London, 1926.

38. Dedekind, R.; Essays on the Theory of Numbers, The Open Court: London, 1901.

39. Cantor, G.; Mathematische Annalen 1895, 46, 481; Cantor, G.; Mathematische Annalen 1897, 49, 207.

40. Silhavý, M.; Archives of Rational Mechanics Analysis 1989, 107, 1. 